\title{
Impact of paravertebral blockade use in geriatric patients undergoing thoracic surgery on postoperative adverse outcomes
}

\author{
Chaoyang Tong ${ }^{1 \#}$, Hongwei Zhu ${ }^{1 \#}$, Bin $\mathrm{Li}^{2}$, Jingxiang $\mathrm{Wu}^{1}$, Meiying $\mathrm{Xu}^{1}$ \\ ${ }^{1}$ Department of Anesthesiology, ${ }^{2}$ Department of Thoracic Surgery, Shanghai Chest Hospital, Shanghai Jiao Tong University, Shanghai 200025, China \\ Contributions: (I) Conception and design: C Tong, H Zhu, M Xu; (II) Administrative support: J Wu, M Xu; (III) Provision of study materials or \\ patients: C Tong, H Zhu, B Li; (IV) Collection and assembly of data: C Tong, H Zhu; (V) Data analysis and interpretation: C Tong, H Zhu, M Xu; (VI) \\ Manuscript writing: All authors; (VII) Final approval of manuscript: All authors. \\ \#These authors contributed equally to this work as co-first authors. \\ Correspondence to: Meiying Xu. Department of Anesthesiology, Shanghai Chest Hospital, Shanghai Jiao Tong University, No. 241 Huaihai Rd. West, \\ Shanghai 200025, China. Email: xmyxk123@163.com.
}

Background: While it is known that thoracic paravertebral blockade (TPVB) could reduce pain undergoing thoracic surgery, it has not been confirmed whether this reduction in pain reduces pulmonary complications in an elderly population.

Methods: We performed a monocentric retrospective analysis for a prospectively collected patients receiving thoracic surgery with or without intraoperative TPVB between November 7, 2018 and April 1, 2019, at Shanghai Chest Hospital. Whether or not to use TPVB depending on anesthesiologists' preference, the chances of harm and benefit of each patients after discussed with their anesthetist. Chest wall resection, bilateral lung resection, conversion to thoracotomy and ipsilateral reoperation were excluded. A total of 154 patients with lung operations were included in the final analysis, 34 of whom received general anesthesia combined with TPVB (GA-TPVB). The primary outcome was the incidence of postoperative pulmonary complications (PPCs). The secondary outcomes were the incidence of cardiovascular and other complications, required analgesia in post anesthesia care unit (PACU), patient controlled analgesia (PCA) pressing frequency in $24 \mathrm{~h}$, chest tube duration, ICU stay and the hospital length of stay (LOS).

Results: The incidence of PPCs undergoing thoracic surgery was about 21.4\% (33/154). Compared with GA, GA-TPVB could reduce the incidence of PPCs (25\% vs. 9\%, $\mathrm{P}=0.042)$, mostly reduce postoperative atelectasis $(19 \%$ vs. $3 \%, \mathrm{P}=0.021)$. TPVB could reduce the rate of required analgesia in PACU, PCA pressing frequency in $24 \mathrm{~h}$ and chest tube duration. However, there were no significant differences on the rate of cardiovascular and other complications, ICU stay and LOS between the two groups $(\mathrm{P}>0.05)$. Multivariable logistic regression analysis identified preoperative DLCO $2 \geq 92 \%(\mathrm{OR}=0.293, \mathrm{P}=0.006$ ), duration of surgery $<75 \mathrm{~min}(\mathrm{OR}=0.278, \mathrm{P}=0.008)$ and $\mathrm{GA}-\mathrm{TPVB}(\mathrm{OR}=0.270, \mathrm{P}=0.048)$ was associated with fewer PPCs.

Conclusions: Our study shows that general anesthesia combined with TPVB may reduce PPCs by reducing postoperative pain in geriatric patients undergoing thoracic surgery compared with general anesthesia alone.

Trial registration: Chinese Clinical Trial Registry number, ChiCTR1800019526. Registered on Nov 7, 2018.

Keywords: Anesthetic methods; thoracic paravertebral blockade (TPVB); thoracic surgery; postoperative pulmonary complications (PPCs)

Submitted Jul 11, 2019. Accepted for publication Nov 19, 2019.

doi: $10.21037 /$ jtd.2019.12.13

View this article at: http://dx.doi.org/10.21037/jtd.2019.12.13

(C) Journal of Thoracic Disease. All rights reserved. 


\section{Introduction}

Postoperative pulmonary complications (PPCs) are one of the common surgical complications after anesthesia, and the main cause are prolonged hospital stay, increased medical costs and mortality (1-4). Especially in lung resection surgery, the incidence of PPCs is $15-37.5 \%$ has become a major concern after surgery $(1,5,6)$. An appropriate postoperative analgesia after thoracic surgery is mandatory to improve the patient's outcome, reduce complications rate, morbidity, hospital cost and length of stay (LOS) (7). Thoracic paravertebral blockade (TPVB), as a safe and effective method of postoperative analgesia, has the same postoperative analgesic effect and could reduce postoperative complications compared with thoracic epidural (8-10) (which was regarded as the gold standard for postoperative analgesia in thoracic surgery). TPVB is superior to intravenous analgesia in providing stable and long-lasting pain control $(7,11)$, and is more conducive to postoperative pulmonary function recovery (12). As thoracic epidural was shown associated with less PPCs in chronic obstructive pulmonary disease (COPD) patients compared with systemic analgesia (13), the impact on PPCs with intraoperative TPVB in geriatric patients remains unknown. We hypothesized that general anesthesia combined with TPVB in geriatric patients could reduce postoperative adverse outcomes compared with general anesthesia alone.

\section{Methods}

\section{Study design and patient population}

We performed a monocentric retrospective analysis for a prospectively collected patients receiving thoracic surgery with or without intraoperative TPVB between November 7, 2018 and April 1, 2019, at Shanghai Chest Hospital. Whether or not to use TPVB depending on anesthesiologists' preference, the chances of harm and benefit of each patient after discussed with their anesthetist. This study was approved by the Institutional Review Board (KS1862) of Shanghai Jiao tong University Shanghai Chest Hospital with the consent of the patient or family member and signed informed consent (Chinese Clinical Trial Registry number, ChiCTR1800019526).

\section{Criteria for inclusion and exclusion}

Inclusion criteria were patients at least 65 years, ASA I-III grade were scheduled for thoracic surgery were selected.
Chest wall resections, bilateral lung resection, conversion to thoracotomy and ipsilateral reoperation were excluded. A total of 154 patients with lung operations were included in the final analysis, 34 of whom received general anesthesia combined with TPVB (GA-TPVB).

\section{Preoperative preparations and anesthesia protocol}

Patients were monitored with electrocardiography, noninvasive blood pressure, pulse oximetry, capnography. General Anesthesia was induced with $0.6 \mu \mathrm{g} / \mathrm{kg}$ sufentanil and a target-controlled infusion of propofol set to a plasma concentration of $4 \mu \mathrm{g} / \mathrm{mL}$, cisatracurium $0.2 \mathrm{mg} / \mathrm{kg}$ was given to facilitate double-lumen bronchial tube intubation. Propofol administration was adjusted to of $2.5 \mu \mathrm{g} / \mathrm{mL}$ and Cisatracurium adjusted to $0.12 \mathrm{mg} / \mathrm{kg} /$ hour, remifentanil adjusted to $0.1 \mu \mathrm{g} / \mathrm{kg} / \mathrm{h}$ in the maintenance period. Invasive blood pressure monitoring was achieved by radial artery cannulation (IBP) and right internal jugular central venous catheterization (CVP). Patients were placed in lateral decubitus. For TPVB, $15 \mathrm{~mL}$ of $0.5 \%$ ropivacaine was given to T4-T5, performed by specific anesthesiologists under ultrasound guidance before operation. Intraoperatively adoption of lung protective ventilation strategies, including low tidal volume $(\leq 8 \mathrm{~mL} / \mathrm{kg})$ ventilation based on ideal body weight, PEEP $=5 \mathrm{cmH}_{2} \mathrm{O}$, lung recruitment and maintenance of airway pressure $<30 \mathrm{cmH}_{2} \mathrm{O}$. Sufentanil $5 \mu \mathrm{g}$ was given before skin incision. Patient controlled analgesia (PCA) pump was used in all patients after operation. The drug used in the pump was sufentanil $1 \mu \mathrm{g} / \mathrm{kg}+$ dezocine $0.4 \mathrm{mg} / \mathrm{kg}$.

\section{Measurements}

The demographic and baseline data, including gender, age, ASA grade, BMI, history of hypertension, Diabetes, cerebral infarction, radiotherapy and chemotherapy, $\mathrm{FEV}_{1} / \mathrm{FVC}$, DLCO $\%$ and preoperative cognitive function assessment. Operative time, surgical procedure (VATS/ thoracotomy/robotic assisted VATS), type of operation (anatomical lobectomy/non-anatomical lobectomy), type of anesthesia (GA/GA-TPVB), intraoperative hypoxemia $\left(\mathrm{SpO}_{2}<90 \%\right.$, lasting for more than 10 minutes), total fluid volume and duration in PACU were also recorded. The primary outcome was the incidence of PPCs (atelectasis, pulmonary infection, respiratory failure). The secondary outcomes were the incidence of cardiovascular and other complications, required analgesia in post anesthesia care 


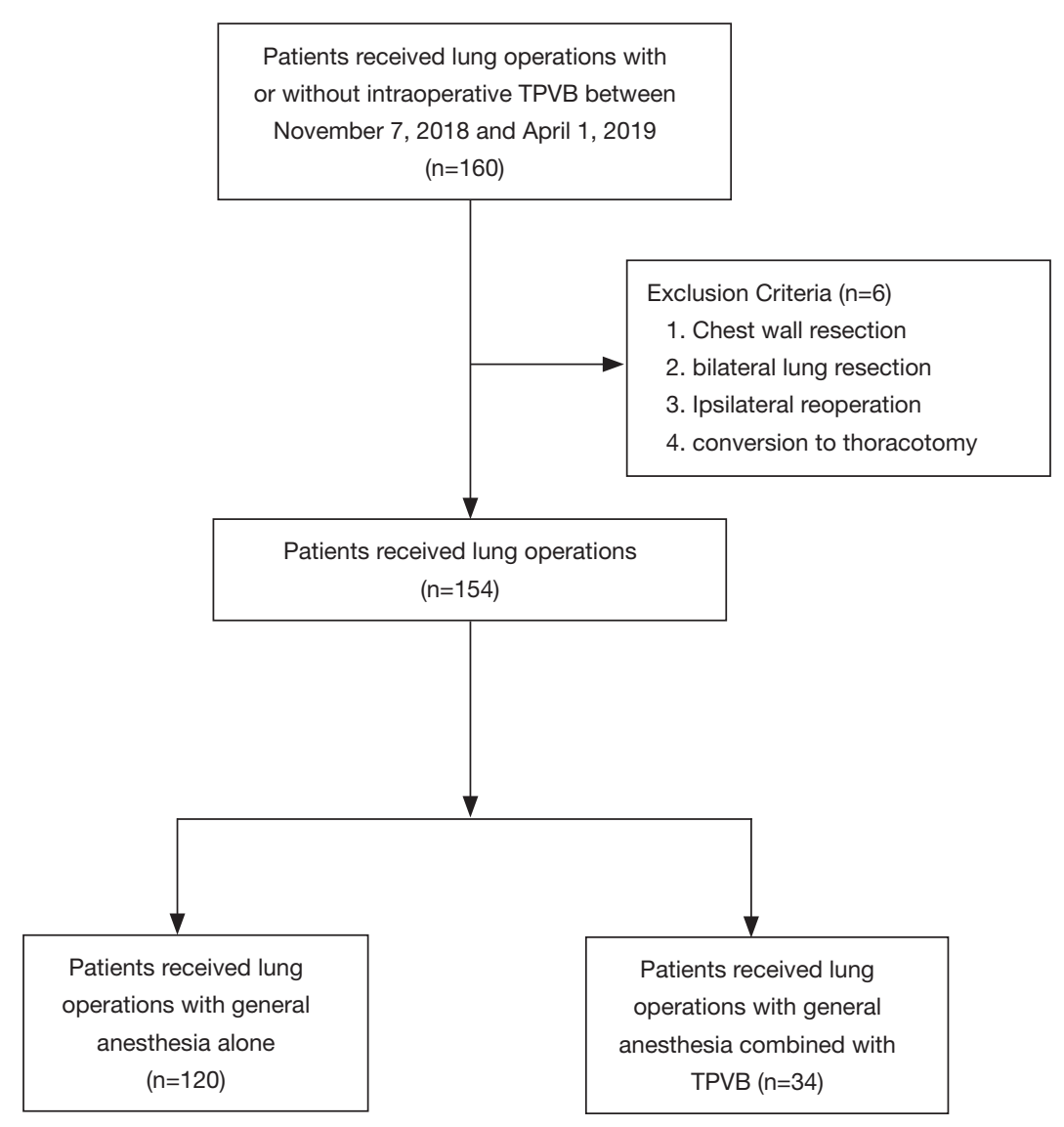

Figure 1 Patient flowchart. TPVB, thoracic paravertebral blockade.

unit (PACU), PCA pressing frequency in $24 \mathrm{~h}$, chest tube duration, ICU stay and the hospital LOS. Atelectasis was measured by radiologists through CXR within 3 days after surgery. Assessment of respiratory failure and pulmonary infection with reference to EPCO (14). All PPCs were evaluated within 7 days after surgery. The extubation criteria were determined by experienced surgeons based on patient's postoperative recovery.

\section{Statistical analysis}

SPSS25.0 statistical software (SPSS Inc., Chicago, IL, USA) was used for data processing. Continuous variables are expressed as mean \pm standard deviation $(\mathrm{SD})$ or median (range) depending on data distribution. Frequency and percentage are used for categorical variables. Two independent sample $t$-test was used to compare the continuous variables with normal distribution. MannWhitney U-test was used to compare continuous variables with non-normal distribution. Chi-square test or Fisher exact test were used to compare categorical variables. We used Kaplan-Meier estimates for chest tube duration, ICU stay and LOS. All risk factors found significantly associated $(\mathrm{P}<0.1)$ with $\mathrm{PPCs}$ by univariate analysis were entered into a multivariable logistic regression model using a forward (LR) selection strategy. The thresholds for DLCO\% and operative time were determined by ROC analysis. $\mathrm{P}<0.05$ was statistically significant.

\section{Results}

A total of 154 patients were enrolled in the final analysis, 34 patients (22\%) who received GA-TPVB (Figure 1). The incidence of PPCs undergoing thoracic surgery was about $21.4 \%$ (33/154). There were no significant differences in pre- and intraoperative patient characteristics between the two groups (Table 1).

Compared with GA, GA-TPVB could reduce the incidence of PPCs (25\% vs. 9\%, $\mathrm{P}=0.042)$, mostly reduce postoperative atelectasis $(19 \%$ vs. $3 \%, \mathrm{P}=0.021)$. TPVB 
Table 1 Pre- and intraoperative patient characteristics

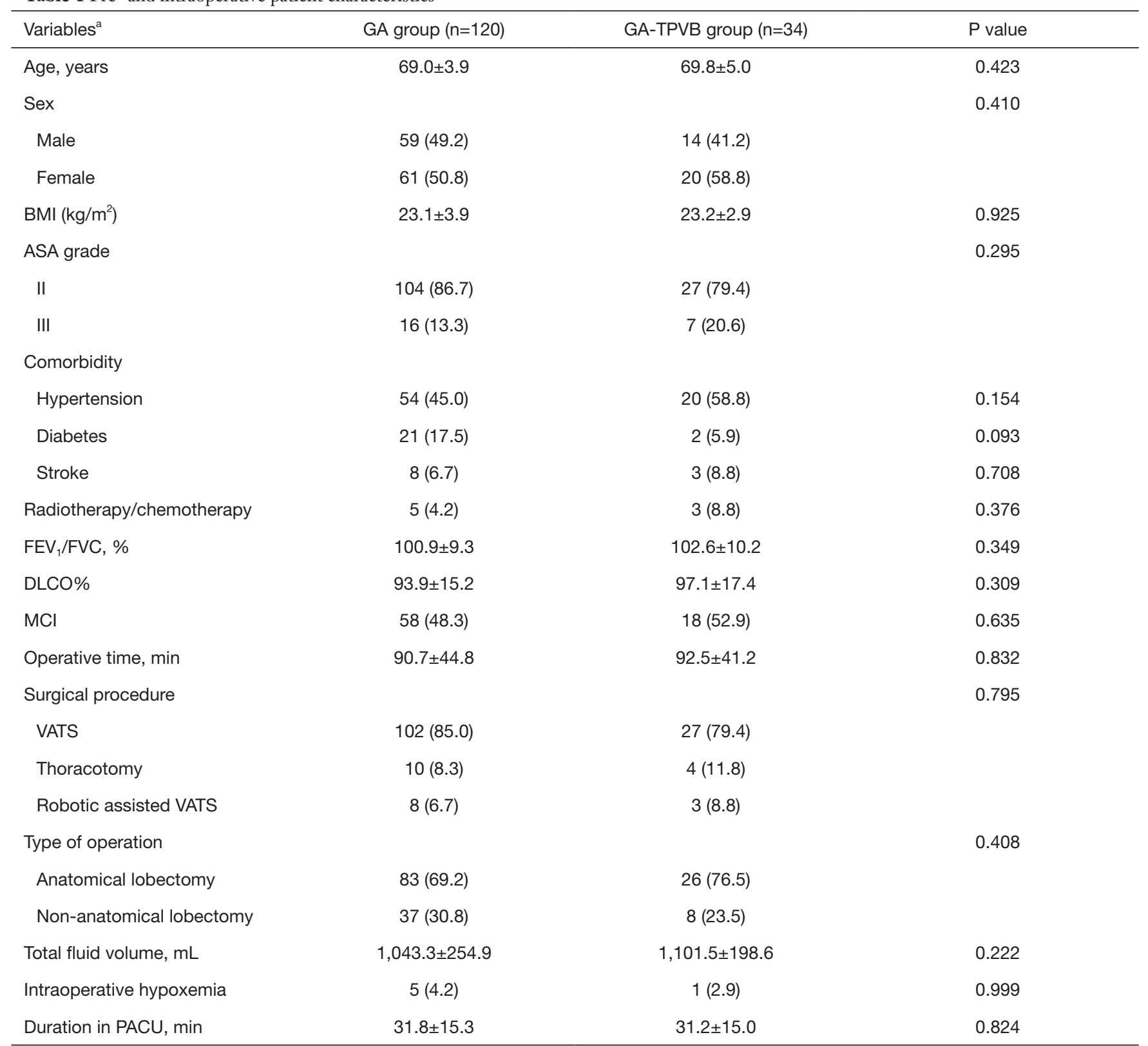

${ }^{a}$, continuous data are shown as mean \pm standard deviation and categorical data as number (\%). Intraoperative hypoxemia: $\mathrm{SpO}_{2}<90 \%$, lasting for more than 10 minutes. BMI, body mass index; ASA, American Society of Anesthesiologists; FEV ${ }_{1}$, forced expiratory volume in 1 second; FVC, forced vital capacity; DLCO, diffusion capacity for carbon monoxide; $\mathrm{MCI}$, mild cognitive impairment; VATS, video-assisted thoracoscopic surgery; PACU, post anesthesia care unit.

could reduce the rate of required analgesia in PACU, PCA pressing frequency in $24 \mathrm{~h}$ and chest tube duration. However, there were no significant differences on the rate of cardiovascular and other complications, ICU stay and LOS between the two groups $(\mathrm{P}>0.05)$ (Table 2).

\section{Univariate and multivariate analysis of potential risk factors for PPCs}

The thresholds for DLCO \% and operative time were determined by ROC analysis, which showed area under the curve values of 0.699 and 0.691 , respectively. Inserting the 
Table 2 Outcomes between GA or GA-TPVB group

\begin{tabular}{lccc}
\hline Variables $^{\text {a }}$ & GA group $(n=120)$ & GA-TPVB group $(n=34)$ & P value \\
\hline PPCs & 30 & 3 & 0.042 \\
Atelectasis & 23 & 1 & 0.021 \\
Pulmonary infection & 9 & 2 & 0.999 \\
Respiratory failure & 4 & 0 & 0.576 \\
Cardiovascular & 10 & 0 & 0.119 \\
Atrial arrhythmia & 8 & 0 & \\
Acute cerebral infarction & 1 & 0 & \\
Myocardial infarction & 1 & 0 & 0.118 \\
Other complications & 1 & 2 & \\
Chylothorax & 0 & 1 & \\
Blood transfusion & 1 & 1 & 0.041 \\
Required analgesia in PACU & 14 & 0 & $<0.001$ \\
PCA pressing frequency in $24 \mathrm{~h}$ & $14 \pm 5$ & $9 \pm 3$ & 0.011 \\
Chest tube duration, day & $4[3-4]$ & $3[2-3]$ & 0.694 \\
Length of stay, day & $4[3-5]$ & $4[3-5]$ & 0.770 \\
ICU stay, day & $1[1-3]$ & $1[1-2]$ & \\
\hline
\end{tabular}

a , continuous data are shown as mean \pm standard deviation and categorical data as number (\%). Chest tube duration, length of stay and ICU stay, values as median (range). ICU, intensive care unit; PPCs, postoperative pulmonary complications; PACU, post anesthesia care unit; PCA, patient controlled analgesia; ICU, intensive care unit.

variables with $\mathrm{P}<0.1$ at univariate analysis in a multivariable logistic regression analysis model, preoperative DLCO \% $\geq 92 \%$ ( OR $=0.293, \mathrm{P}=0.006)$, duration of surgery $<75 \min (\mathrm{OR}=0.278, \mathrm{P}=0.008)$ and $\mathrm{GA}-\mathrm{TPVB}(\mathrm{OR}=0.270$, $\mathrm{P}=0.048$ ) was associated with fewer PPCs (Table 3).

\section{Discussion}

Our study showed that GA-TPVB in geriatric patients undergoing thoracic surgery was associated with fewer PPCs compared with GA. TPVB could reduce the rate of required analgesia in PACU, PCA pressing frequency in $24 \mathrm{~h}$ and chest tube duration. However, based on the data of this study, the effects of GA-TPVB on cardiovascular and other complications, ICU stay and LOS cannot be obtained. Perioperative TPVB use may help to provide interventions for patients at greatest risk of major adverse outcomes after surgery, so interventions can be targeted to those most likely to benefit.

That about 21.4\% (33/154) geriatric patients scheduled for thoracic surgery have probable PPCs is not surprising given the same prevalence of PPCs in other clinical studies $(5,6)$. For instance, depending upon definition of PPCs and type of surgery, the prevalence of PPCs ranges from $9 \%$ to $40 \%$ (15-18). The definition of PPCs in this study is based on the European Perioperative Clinical Outcome definition (14), which is determined by experienced anesthesiologists and surgeons with relative reliability. Moreover, TPVB is performed by experienced anesthesiologists under the guidance of ultrasound, which could ensure the effectiveness and safety of postoperative analgesia. Furthermore, this study is not limited to one type of thoracic surgery (including VATS/thoracotomy/robotic assisted VATS), so our study is applicable to a wider range of elderly patients undergoing elective thoracic surgery.

In our study, TPVB could reduce the rate of required analgesia in PACU, PCA pressing frequency in $24 \mathrm{~h}$ and chest tube duration. The mechanism probably by which GA-TPVB reduces the incidence of PPCs may be to provide stable and long-lasting pain control after surgery to 
Table 3 Univariate ${ }^{a}$ and multivariate analysis of potential risk factors for PPCs

\begin{tabular}{|c|c|c|c|c|c|}
\hline Variable & \multicolumn{2}{|c|}{ PPCs } & $\frac{\text { Univariate }}{\mathrm{P} \text { value }}$ & \multicolumn{2}{|c|}{ Multivariate } \\
\hline $\mathrm{BMI}\left(\mathrm{kg} / \mathrm{m}^{2}\right)$ & $23.5 \pm 2.8$ & $21.8 \pm 5.7$ & 0.018 & & \\
\hline DLCO\% & & & 0.003 & & \\
\hline$<92 \%$ (reference) & 51 & 23 & & & \\
\hline Anesthesia methods & & & 0.042 & & \\
\hline GA (reference) & 90 & 30 & & & \\
\hline GA-TPVB & 31 & 3 & & $0.270(0.074-0.991)$ & 0.048 \\
\hline Operative time (min) & & & 0.007 & & \\
\hline Intra-hypoxemia & 2 & 4 & 0.019 & & \\
\hline
\end{tabular}

a, Hosmer-Lemeshow test $\mathrm{P}=0.532$; C statistic: 0.741 . Cl, confidence interval; OR, odds ratio; BMI, body mass index; DLCO, diffusion capacity for carbon monoxide; PPCs, postoperative pulmonary complications; GA-TPVB, general anesthesia combined with thoracic paravertebral block.

promote recovery of lung function $(7,11)$. While poor pain control after surgery which do not allow the patients to take deep breath and ultimately lead to atelectasis and retention of secretions $(7,19,20)$.

Protective lung ventilation (PLV) during one-lung ventilation (OLV) for thoracic surgery is frequently recommended to reduce pulmonary complications (21). However, the effect of anesthesia methods on PPCs has been controversial, especially in regional anesthesia. Powell et al. (22) compared the effects of TPVB and epidural blockade on postoperative adverse outcomes after pneumonectomy and concluded that the increased incidence of major PPCs was associated with thoracic epidural block, but did not prolong the hospital length of day. A retrospective propensity score-matched study by Blackshaw et al. (23) found that TPVB did not reduce the incidence of PPCs, other postoperative complications, morbidity and mortality and prolong postoperative hospital stay compared with epidural block in open lung resection which were consistent with randomised controlled trials and systematic reviews $(24,25)$. Thoracic epidural analgesia was associated with reduced PPCs and mortality in patients with severe COPD compared with systemic analgesia (13). Therefore, we infer that TPVB use in geriatric patients could reduce PPCs after surgery compared with general anesthesia alone, and the results of this study also confirm this. There were no significant differences in rates of other postoperative complications, ICU stay and LOS based on this study.

Previous studies have identified risk factors of PPCs, including increased age, gender, BMI $\geq 30 \mathrm{~kg} / \mathrm{m}^{2}$, smoking, ASA score $\geq 3$, preoperative $\mathrm{FEV}_{1} \leq 60 \%$, COPD, duration of surgery, open surgery and increased intraoperative fluid administration (26-32). In our study, multivariable logistic regression analysis also showed that preoperative DLCO\% $<92 \%$, duration of surgery $\geq 75 \mathrm{~min}$ and general anesthesia alone were independent risk factors for PPCs. All of these modifiable risk factors should be of concern to clinicians to minimize the incidence of PPCs after thoracic surgery.

This study also has certain limitations. It has the same bias as any secondary analysis of data. Also, we were unable to directly compare analgesic efficacy due to incomplete data. Finally, since the patients were not randomized into receiving GA or GA + TPVB. The biases introduced by this decision may have an impact on PPCs.

\section{Conclusions}

Our study shows that general anesthesia combined with TPVB may reduce PPCs by reducing postoperative pain in geriatric patients undergoing thoracic surgery compared 
with general anesthesia alone.

\section{Acknowledgments}

The authors would like to thank the nurse anesthetists in the operating room of the Shanghai Chest Hospital, Shanghai, People' republic of China, for their involvement and support.

Funding: This work was supported by Shanghai Municipal Science and Technology Committee (Western Medicine Guided Project) 16411967600 Fund and Shanghai Municipal Commission of Health and Family Planning Project (201840319).

\section{Footnote}

Conflicts of Interest: The authors have no conflicts of interest to declare.

Etbical Statement: The authors are accountable for all aspects of the work in ensuring that questions related to the accuracy or integrity of any part of the work are appropriately investigated and resolved. This study was approved by the Institutional Review Board (KS1862) of Shanghai Jiao tong University Shanghai Chest Hospital with the consent of the patient or family member and signed informed consent.

\section{References}

1. Canet J, Gallart L, Gomar C, et al. Prediction of postoperative pulmonary complications in a populationbased surgical cohort. Anesthesiology 2010;113:1338-50.

2. Shapiro M, Swanson SJ, Wright CD, et al. Predictors of major morbidity and mortality after pneumonectomy utilizing the Society for Thoracic Surgeons General Thoracic Surgery Database. Ann Thorac Surg 2010;90:927-34.

3. Moonesinghe SR, Harris S, Mythen MG, et al. Survival after postoperative morbidity: a longitudinal observational cohort study. Br J Anaesth 2014;113:977-84.

4. Pearse RM, Moreno RP, Bauer P, et al. Mortality after surgery in Europe: a 7 day cohort study. Lancet 2012;380:1059-65.

5. Agostini P, Cieslik H, Rathinam S, et al. Postoperative pulmonary complications following thoracic surgery: are there any modifiable risk factors? Thorax 2010;65:815-8.

6. Mazo V, Sabaté S, Canet J, et al. Prospective external validation of a predictive score for postoperative pulmonary complications. Anesthesiology 2014;121:219-31.

7. Piraccini E, Pretto EA Jr, Corso RM, et al. Analgesia for thoracic surgery: the role of paravertebral block. HSR Proc Intensive Care Cardiovasc Anesth 2011;3:157.

8. Davies RG, Myles PS, Graham JM. A comparison of the analgesic efficacy and side-effects of paravertebral vs. epidural blockade for thoracotomy--a systematic review and meta-analysis of randomized trials. Br J Anaesth 2006;96:418-26.

9. Baidya DK, Khanna P, Maitra S. Analgesic efficacy and safety of thoracic paravertebral and epidural analgesia for thoracic surgery: a systematic review and meta-analysis. Interact Cardiovasc Thorac Surg 2014;18:626-35.

10. Kosiński S, Fryźlewicz E, Wiłkojć M, et al. Comparison of continuous epidural block and continuous paravertebral block in postoperative analgaesia after video-assisted thoracoscopic surgery lobectomy: a randomised, noninferiority trial. Anaesthesiol Intensive Ther 2016;48:280-7.

11. Karmakar MK. Thoracic paravertebral block. Atlas of Ultrasound-Guided Regional Anesthesia. Elsevier, 2019:286-315.

12. Matyal R, Montealegre-Gallegos M, Shnider M, et al. Preemptive ultrasound-guided paravertebral block and immediate postoperative lung function. Gen Thorac Cardiovasc Surg 2015;63:43-8.

13. Elsayed H, McKevith J, McShane J, et al. Thoracic epidural or paravertebral catheter for analgesia after lung resection: is the outcome different? J Cardiothorac Vasc Anesth 2012;26:78-82.

14. Jammer I, Wickboldt N, Sander M, et al. Standards for definitions and use of outcome measures for clinical effectiveness research in perioperative medicine: European Perioperative Clinical Outcome (EPCO) definitions: A statement from the ESA-ESICM joint taskforce on perioperative outcome measures. Eur J Anaesthesiol 2015;32:88-105.

15. Ackland GL, Iqbal S, Paredes LG, et al. Individualised oxygen delivery targeted haemodynamic therapy in highrisk surgical patients: a multicentre, randomised, doubleblind, controlled, mechanistic trial. The Lancet Respir Med 2015;3:33-41.

16. Warner DO. Preventing Postoperative Pulmonary Complications: The Role of the Anesthesiologist. Anesthesiology 2000;92:1467-72.

17. Arozullah AM, Daley J, Henderson WG, et al. Multifactorial risk index for predicting postoperative 
respiratory failure in men after major noncardiac surgery. Ann Surg 2000;232:242.

18. Ireland CJ, Chapman TM, Mathew SF, et al. Continuous positive airway pressure (CPAP) during the postoperative period for prevention of postoperative morbidity and mortality following major abdominal surgery. Cochrane Database Syst Rev 2014;(8):CD008930.

19. Xue FS, Li BW, Zhang GS, et al. The influence of surgical sites on early postoperative hypoxemia in adults undergoing elective surgery. Anesth Analg 1999;88:213-9.

20. Sabanathan S, Eng J, Mearns AJ. Alterations in respiratory mechanics following thoracotomy. J R Coll Surg Edinb 1990;35:144-50

21. Petrucci N, De Feo C. Lung protective ventilation strategy for the acute respiratory distress syndrome. Cochrane Database Syst Rev 2013;(2):CD003844.

22. Powell ES, Cook D, Pearce AC, et al. A prospective, multicentre, observational cohort study of analgesia and outcome after pneumonectomy. Br J Anaesth 2011;106:364-70.

23. Blackshaw WJ, Bhawnani A, Pennefather SH, et al. Propensity score-matched outcomes after thoracic epidural or paravertebral analgesia for thoracotomy. Anaesthesia 2018;73:444-9.

24. Joshi GP, Bonnet F, Shah R, et al. A systematic review of randomized trials evaluating regional techniques for postthoracotomy analgesia. Anesth Analg 2008;107:1026-40.

25. Gulbahar G, Kocer B, Muratli SN, et al. A comparison of epidural and paravertebral catheterisation techniques in post-thoracotomy pain management. Eur J Cardiothorac

Cite this article as: Tong C, Zhu H, Li B, Wu J, Xu M. Impact of paravertebral blockade use in geriatric patients undergoing thoracic surgery on postoperative adverse outcomes. J Thorac Dis 2019;11(12):5169-5176. doi: 10.21037/jtd.2019.12.13
Surg 2010;37:467-72.

26. Agostini PJ, Lugg ST, Adams K, et al. Risk factors and short-term outcomes of postoperative pulmonary complications after VATS lobectomy. J Cardiothorac Surg 2018;13:28.

27. Kaufmann KB, Loop T, Heinrich S, et al. Risk factors for post-operative pulmonary complications in lung cancer patients after video-assisted thoracoscopic lung resection: Results of the German Thorax Registry. Acta Anaesthesiol Scand 2019;63:1009-18.

28. Agostini P, Cieslik H, Rathinam S, et al. Postoperative pulmonary complications following thoracic surgery: are there any modifiable risk factors? Thorax 2010;65:815-8.

29. Arslantas MK, Kara HV, Tuncer BB, et al. Effect of the amount of intraoperative fluid administration on postoperative pulmonary complications following anatomic lung resections. J Thorac Cardiovasc Surg 2015;149:314-20.

30. Traibi A, Grigoroiu M, Boulitrop C, et al. Predictive factors for complications of anatomical pulmonary segmentectomies. Interact CardioVasc Thorac Surg 2013;17:838-44.

31. Yang J, Xia Y, Yang Y, et al. Risk factors for major adverse events of video-assisted thoracic surgery lobectomy for lung cancer. Int J Med Sci 2014; 11:863.

32. Sandri A, Papagiannopoulos K, Milton R, et al. Highrisk patients and postoperative complications following video-assisted thoracic surgery lobectomy: a case-matched comparison with lower-risk counterparts. Interact CardioVasc Thorac Surg 2015;21:761-5. 